\title{
Reconstrucción histórica de la formación de profesores de ciencias en colombia. El título porfesional de licenciado
}

\author{
Historical reconstruction of science teachers education in colombia. \\ Rómulo Gallego Badillo', Royman Pérez Miranda ${ }^{1}$ e Iván Rincón Pabón ${ }^{2}$
}

${ }^{1}$ Profesores de la Universidad Pedagógica Nacional. Bogotá, D. C. Colombia. ${ }^{2}$ Asesor técnico del Grupo. Grupo de Investigación Representaciones y Conceptos Científicos - Grupo IREC. rgallego@pedagogica.edu.co royman@pedagógica.edu.co

\section{Resumen}

En esta ponencia se resumen los resultados de un proyecto de investigación evaluado y apoyado por el sistema de investigación de la Universidad Pedagógica Nacional, en el cual se hizo un estudio basado en el análisis tanto de fuentes primarias, como secundarias, sobre la creación, desarrollo y desaparición de la Escuela Normal Superior de Colombia (ENSC), como el proyecto político y educativo que persiguió en Colombia, en los albores del siglo XX, una educación laica para todos. Es en esta Institución y en un programa de formación de profesores que por primera vez se empiezan a enseñar en el país las ciencias por sí mismas. Se presenta, en consecuencia, una reconstrucción histórica.

Palabras claves

Historia, epistemología, didáctica, formación de profesores de ciencias.

\section{Abstract}

This paper summarizes the results of a research project evaluated and supported by the research system of Pedagógica Nacional University, which carried out a study based on the analysis of both primary and secondary sources on the creation, development and disappearance of the Escuela Normal Superior de Colombia (ENSC), as educational and political project pursued in Colombia, at the early twentieth century. The historical reconstruction is presented, due to the fact that this institution and its teachers training program were the pioneers in teaching science.

\section{Keywords}

History, science philosophy, Didactics, science teachers' education.

\section{Introducción}

La primera preocupación por la formación de profesores, en este caso de la química, se debe a E. Frankand (1825 - 1899) quien en 1870 fundó la primera escuela de verano con esta intención (Brock, 1998). En general, el proyecto político y educativo para llevar las ciencias de la naturaleza a los niveles primario y secundario de los sistemas educativos es de finales del siglo XIX y comienzos del XX (Calatayud, Carbonell, Carrascosa, Furió Mas, Gil Pérez, Grima, Hemández, Yopis, Martínez Torregrosa, Payá, Ribó, Satoca, Solbes, Soler, y Vildhes, 1986); con dicho proyecto empezó a surgir el problema de la formación de profesores de ciencias y la creación de instituciones con esta finalidad. Anótese que la enseñanza de las ciencias y la redacción de libros didácticos, tanto como la realización de experimentos para el gran público, fue parte de la denominada institucionalización 
de las ciencias, sobre todo durante el período conocido como el de la revolución científica (Bowler y Morus, 2007).

Por otro lado, hay también que señalar que entre 1830 y 1840, A. Comte desarrolló su propuesta acerca de la aproximación positivista, que se constituyó en la filosofía del conocimiento cientúico y del método científico, hasta 1937, auando el profesor K. Popper, con la publicación de su libro "Logik der Forschung", da comienzo a la denominada era pospositivista (Laudan, 2005). En Colombia, cualquier discusión acerca del positivismo fue oficialmente prohibida a finales del siglo XIX y en su lugar se estableció el Neotomismo en cabeza de Monseñor J. M. Carrasquilla desde su cátedra en el Colegio Mayor del Rosario (Jaramillo, 1986). En la ENSC, se intentó introducir en el método científico a quienes se formaban inicialmente como profesores, para lo aual se estableció una asignatura de filosofía (Socarrás, 1987), que no se ocupó precisamente de la filosofía de las ciencias.

\section{El contexto educativo de la enseñanza de las ciencias en el país}

En Colombia quienes enseñaban ciencias en secundaria, eran religiosos en activo o que habían colgado sus hábitos. Se dice que leían frente a los estudiantes los contenidos de los textos de enseñanza y auando el tema no era del dominio de estos profesores cerraban el libro y hacían que los estudiantes anotaran en sus cuadernos que "eso no tocaba" (Amado y Rodríguez, 1988). Fungían también como enseñantes los hijos díscolos de las dases locales dominantes (Mora, 1996), designados merced al tráfico de influencias políticas. Por otro lado e igualmente, se desempeñaban como docentes de ciencias, ingenieros y médicos, quienes lo hacían en aquellas horas que les dejaban libres sus ocupaciones propiamente profesionales; tomaban las cátedras en la perspectiva de un ingreso monetario adicional.

Quienes se han ocupado de historiar el desarrollo de la educación en el país, como se demostrará posteriormente, en los resultados de sus investigaciones destacan cómo esta se centró exdusivamente en la formación de abogados, ayyos programas académicos carecían de rigor metodológico y en el que cabían aualesquiera disertaciones, todo en el interior de un diletantismo supuestamente indispensable para el ejercicio profesional que requería de este bagaje, con miras a obtener éxito en los estrados judiciales. Todos los esfuerzos que se hicieron para enseñar ciencias a la juventud colombiana en esa segunda mitad del siglo XIX fracasaron. Induso, la sociedad bogotana tildó a esas ciencias de la naturaleza como inútiles (Melo, 1986; Jaramillo, 1984).

En un número de la Revista de Educación, de 1935, órgano de la Dirección de Educación del Departamento de Norte de Santander, ya desaparecida, se destaca que los profesores de este nivel son simples aficionados que solo persiguen con esta ocupación mejorar sus ingresos económicos. Igualmente referencia un artículo del periódico El Tiempo, del 6 de febrero de 1936, en el que se informa sobre los resultados de los exámenes de revisión establecidos por el Ministerio de Instrucción Pública de ese entonces, que el $50 \%$ de los bachilleres no manejan el concepto de porcentaje y que, además, han comprado el tútulo correspondiente. Se subraya el fracaso de la educación secundaria y el hecho de que los establecimientos no cuenten con los materiales indispensables para la enseñanza ni con el personal idóneo para encargarse de las cátedras (Mora, 1996).

El 9 de febrero de 1930, después de aurenta años de hegemonía del partido político conservador colombiano, accede a la presidencia de la república el liberal Enrique Olaya Herrera, quien propone la necesidad de otorgarle derechos civiles a la mujer, elevar el nivel de vida del campesinado colombiano y formula una política de puertas abiertas al capital extranjero. Al iniciar su gobiemo, el analfabetismo llega al $63 \%$ en infantes en edad escolar, así como es notoria la escasez de escuelas normales y ostensible un bajo nivel de preparación de los educadores. Lo 
anterior, entre otros, se constituye en uno de los fundamentos para la creación en Colombia de la ENSC. Al comenzar el siglo XX la mayoría de la población nacional es analfabeta y se debate en las más lamentables condiciones de nutrición y salubridad; algo sobre lo cual había que tomar medidas urgentes si se le quería salir al paso a una modemización del país. Se propuso entonces la capacitación docente y con este propósito se fundó la Facultad de Educación de la Universidad Nacional de Colombia.

Es en este contexto en el que en el panorama legal y educativo del país se crea la ENSC en Bogotá. La República Liberal, encabezada por el Presidente López Pumarejo, mediante la Ley 39 de 1936 (Febrero 21) "por la cual se crea en el Ministerio de Educación Nacional la Sección de Publicaciones, se autoriza el envío de profesores universitarios al Exterior y se dictan otras disposiciones en el ramo de la Educación", se dispone en el "Artículo $4^{\circ}$ que: La Facultad de Ciencias de la Educación continuará funcionando con el nombre de Escuela Normal Superior bajo la dirección inmediata del Gobiemo y con independencia de la Universidad Nacional, no obstante lo dispuesto en la Ley 68 de 1935".

La ENSC inició sus labores académicas en 1937 y fue solo en sus primeros años de funcionamiento en los que cosechó todo el renombre por el aual se le recuerda; los restantes fueron de real decadencia (Ocampo, 1978), dada la persecución que se instauró contra ella y el estrangulamiento financiero al que fue sometida, lo que causó que profesores y estudiantes desplazaran su interés a la lucha por defenderla. Una de las tantas razones que se adujeron en su contra, aludía a los problemas éticos y morales que significaba el hecho de que jóvenes y señoritas compartieran los mismos espacios escolares.

Bajo el Gobiemo del presidente conservador, Laureano Gómez, la ENSC, es escindida, mediante el Decreto distinguido con el número 019 de 1951 (Enero 30), "...por el cual se adopta el plan de estudios para los Institutos de Enseñanza Pedagógica y se dictan otras disposiciones sobre Educación Normalista", todo esto dentro de las facultades otorgadas por el Artículo 121 de la Constitución de 1886, que estableáa el estado de sitio o de excepción; el Decreto 019 aludido, disponía en su artículo 13 que "A partir de la vigencia de este Decreto la Escuela Normal Superior de Bogotá llevará el nombre de Escuela Normal Universitaria, y las Esavelas Normales Regulares Nacionales, el de Escuelas Normales Superiores".

Los jóvenes serán, entonces, trasladados a la ciudad de Tunja, capital del Departamento de Boyacá, a la Escuela Normal Universitaria para varones, de la que surgirá la Universidad Pedagógica de Colombia, hoy Universidad Pedagógica y Tecnológica de Colombia. Las señonitas se quedarán en Bogotá, en la Escuela Normal Universitaria Femenina, que luego pasará a ser la Universidad Pedagógica Femenina y, finalmente, la Universidad Pedagógica Nacional.

\section{La creación del título profesional de licenciado}

El título de "Licenciado" es el más antiguo y de mayor prosapia: se inventa en la Universidad de Paris, en la Edad Media, para garantizarle a la comunidad la idoneidad de los egresados de sus tres facultades históricas. La de teología otorgaba el tútulo de "Litentia doscendi"; la de medicina el de "Litentia operandis"; y la de derecho el de "Litentia judicandis" (Gallego Badillo, Pérez Miranda, Torres de Gallego y Amador Rodríguez, 2004).

En 1934, con el Decreto 1379, en el gobiemo de de Enrique Olaya Herrera en Facultad de Educación adscrita a la Universidad Nacional de Colombia dentro de los programas existentes para la formación de profesores, manda que se les otorgue a los egresados el título de Licenciados. Con el Decreto 2178 de 1930 establece el "Curso de Estudios Superiores para Señoritas" en el Instituto Pedagógico Nacional (IPN) en Bogotá. Con el también Decreto 850 de 1934, se crea la Facultad de 
Educación para mujeres, dependiendo del IPN e igualmente como parte de la Universidad Nacional de Colombia, también se les otorga el tútulo de "Licenciados". En el gobierno del presidente liberal Alfonso López Pumarejo, el de la "Revolución en marchd", se optó en 1935, a través del Decreto 1917 y mediante la Ley 39 de 1936, por crear la ENSC, en la cual se conservó el título de Licenciado.

\section{La atmósfera hostíl contra la profesión de licenciado}

Los primeros egresados de la ENSC empezaron a desplazar del ejercicio docente a los sacerdotes, los abogados, los médicos y los ingenieros en los colegios de secundaria, dando inició a una campaña de descrédito contra la profesión de Licenciado. Se acudió entonces al viejo paradigma de los maestros artesanos de la Edad Media que rezaba que para enseñar el oficio, había que dominarlo. Se invocó el hecho curricular del sacrificio y la limitación de la extensión de los contenidos curriculares en ciencias, en aras de aquellos que tenían que ver, necesariamente, con ese nuevo ejercicio profesional que se había inventado: el del licenciado.

Esos profesionales que ejercían en las horas que les dejaban disponibles sus propias ocupaciones profesionales, más el también desplazamiento como educadores de aquellos hijos "díscolos" de las familias pudientes en las regiones, se sumaron a la campaña de desprestigio que se organizó en contra de la ENSC y sus egresados. Con base en una no historia social de la educación en ciencias en Colombia, aún mucho tiempo después de desaparecida la ENSC, todavía en la segunda mitad del siglo XX, se acusaba a los licenciados en Biología y Química de ser los responsables de que entre la población colombiana siguiera existiendo una baja estima por las ciencias de la naturaleza en general, y por la química en particular (Villaveces, 1990).

No obstante, es necesario inscribir históricamente esta reacción, por cuanto la formación inicial de profesores de ciencias en Colombia nació, para reiterarlo, dentro de un proyecto político y educativo que persiguió introducir a la nación en una educación laica y gratuita para todos; una esperanza que aun gravita en la conformación de la nacionalidad esperada. El horizonte de sentido parece haber sido siempre educar a la población en esa racionalidad no absolutista a partir de la cual es factible comprender el desarrollo histórico de cada una de las ciencias de la naturaleza (Estany, 2005), labor ardua en un país educado históricamente, desde la época colonial, en el interior de una concepción totalmente dogmática, en el cual el mundo se halla dividido entre buenos y malos; "maniqueísmo" este, que termina ordinariamente en sanciones oficiales.

De hecho, en el interior del Ministerio de Educación Nacional, los técnicos en didáctica y en pedagogía, en este caso de las ciencias, como consecuencia de sus posibles desconocimientos de la institucionalización de la actividad cient́fica en el siglo XVII y de los resultados de esta educación iniciándose el siglo XX, apoyando perspectivas políticas cuyas motivaciones habría que develar en su historia, decidieron crear un concurso para la vinculación de profesores procedentes de otros programas académicos de formación. Con los fundamentos establecidos por el poder político, en Colombia, de un plumazo gubemamental, se dejó de lado toda la historia de la constitución de la didáctica de las ciencias como una disciplina conceptual y metodológicamente fundamentada.

De cualquier manera, mirando críticamente la historia, son los Licenciados en ciencias, egresados de ENSC, quienes llevan cabo el trabajo pedagógico y didáctico, de socializar entre la población estudiantil colombiana la versión de actividad cient́fica dominante de la época. Fueron ellos quienes se dedicaron a escribir los primeros textos de enseñanza de las ciencias, textos de los que carecía el país (Socarrás, 1987). Se enfrentaron a unas condiciones ideológicas y culturales para las que los contenidos de las ciencias de la naturaleza eran contrarios a las concepciones de mundo enseñados por los representantes de la Iglesia Católica colombiana. 
Otro aspecto por considerar es el pensar que el relativo retraso científico y tecnológico del país se deba a que el gobiemo liberal de esas primeras décadas del siglo $X X$, haya invertido todos sus esfuerzos en la formación de profesores de ciencias y no en el apoyo de la investigación (Puerta, 1989); una convicción, para reiterarlo, que se halla por fuera de las investigaciones en la historia social de las ciencias, para la que la actividad de enseñanza y la formación de profesores ha sido crucial en el proceso de institucionalización y acogida social del pensamiento y de la actividad cientúfica.

\section{Conclusiones}

Por las razones aquí recopiladas, Colombia ha desestimado considerablemente a lo largo del siglo XX la labor desempeñada por sus licenciados en ciencias, tan ciiticable como se quiera esa formación, ha incidido positivamente en el proceso de socialización del conocimiento cient́fico entre la población nacional. De ahí que estas razones se agreguen a las que dan cuenta del retraso cientúfico y tecnológico en el que se encuentra el país, como lo muestran varios indicadores.

En efecto, José Luís Barragán Duarte en el artículo tïtulado "Universidades colombianas: ¿Invisibles en la red?", en "UNperiódicd" No. 109, del Domingo 9 de Marzo de 2008, apoyado en los datos del "Ranking Web" de universidades en el mundo" muestra para el caso latinoamericano, que la Universidad Autónoma de México ocupa el puesto 59, la Universidad de São Paulo el 114, la Universidad de Campinas el 197, la Universidad de Chile el 210, la Universidad Federal de Santa Catarina (Brasil) el 234, la Universidad de Buenos Aires el 361, la Universidad Federal de Río de Janeiro el 377, la Universidad Federal de Río Grande del Sur el 396, el Instituto Tecnológico de Monterrey (México) el 421 y la Pontificia Universidad Católica de Chile el 505.

De la misma manera, muestra los puestos ocupados por algunas universidades colombianas, así: la Universidad de los Andes el 873, la Universidad Nacional de Colombia el 899, la Universidad de Antioquia el 961, la Universidad del Valle el 1146, la Pontificia Universidad Javeriana el 1297, la Universidad del Cauca el 1491, la Universidad Nacional de Colombia sede de Medellín el 2145, la Universidad Tecnológica de Pereira el 2315 y la Escuela de Administración, Finanzas y Tecnologías (Medellín) el 2334. Aún no aparece en el "Ranking" la Universidad Industrial de Santander y mucho menos el resto de las que en el país ostentan el nombre de universidad. Se celebra que las "ranquedas" aparezcan, no obstante a los interesados se les sugiere realizar las operaciones aritméticas para obtener la diferencia que separa a estas universidades colombianas de, por ejemplo, la Pontifica Universidad Católica de Chile y, por qué no, de la Universidad Autónoma de México.

Una adaración es indispensable. Para esta dasificación se tienen en cuenta, entre otros factores, el número de Premios Nobel que ejercen como profesores, el número de artículos producto de investigación publicados en revistas especializadas, arbitradas e indexadas, las citas que otros investigadores hacen de esos artículos en revistas diferentes y de las mismas calidades, las patentes obtenidas, en otras palabras de las contribuciones de los grupos de investigación de las universidades a la producción de conocimiento cient́fico y tecnológico. Se induyen, además, aspectos estrictamente curriculares como el número de programas de posgrado, los objetivos de los mismos, las condiciones de acreditación oficial de dichos programas, el número de alumnos inscritos en estos programas y los porcentajes de los que obtienen finalmente el título correspondiente.

En la búsqueda de una explicación del relativo rezago de las universidades colombianas en relación con las latinoamericanas, el profesor Marcos Palacios en un artículo que tituló "Rezago grave" en ID. Lecturas de Fin de Semana, del periódico El Tiempo de Bogotá, de Octubre 6 de 2007, además del análisis comparativo de los reaursos financieros que en términos del porcentaje 
del Producto Interno Bruto el gobiemo de Colombia dedica a apoyar la investigación científica y tecnológica, señala que una explicación de ese rezago se encontranía en el hecho de que en Latinoamérica el sistema educativo del país fue el último en adoptar una concepción laica de las ciencias.

\section{Bibliografia}

Brock, W. H. (1998). Historia de la química. Madrid: Alianza.

Bowler, P. J. y Morus, I. (2007). Panorama general de la ciencia modema. Barcelona: Crítica.

Jaramillo, R. (1986). Introducción de la filosofía modema en Colombia. Universidad y Sociedad. En Argumentos, Nos. 14/15, 16/17.

Mora, J. (1996). Los conflictos educativos entre la Iglesia y el Estado. Primer gobiemo de López Pumarejo 1934 - 1938. Bogotá: Universidad Antonio Nariño. 